\title{
Maternal inflammatory markers for chorioamnionitis in preterm prelabour rupture of membranes: a systematic review and meta-analysis of diagnostic test accuracy studies
}

\author{
Angela Koech Etyang $^{1 *}$ (D), Geoffrey Omuse ${ }^{2}$, Abraham Mwaniki Mukaindo ${ }^{1}$ and Marleen Temmerman ${ }^{1}$
}

\begin{abstract}
Background: There is no consensus on the role of inflammatory markers in identifying chorioamnionitis in preterm prelabour rupture of membranes (PPROM). We set out to evaluate the accuracy of maternal blood C-reactive protein (CRP), procalcitonin and interleukin 6 (IL6) in diagnosis of histological chorioamnionitis and/or funisitis (HCA/Funisitis) in PPROM.

Methods: We searched MEDLINE, EMBASE and The Cochrane Library from inception to January 2020 for studies where maternal blood CRP, procalcitonin or IL6 was assessed against a reference standard of HCA/Funisitis in PPROM. The Quality Assessment of Diagnostic Accuracy Studies 2 (QUADAS-2) tool was used to assess methodological quality. Hierarchical summary receiver operating characteristic (SROC) models were used to construct summary curves. Bivariate models were used to obtain summary estimates for studies with the same cut-off.

Results: We included 23 studies reporting HCA/Funisitis in 902 of 1717 women, median prevalence 50\% (inter-quartile range 38-57). Of these studies, 20 were prospective cohort design and 3 were retrospective cohort. Eleven studies reported the index test against a reference standard of HCA and/or funisitis, 10 reported HCA alone and 2 reported funisitis alone. Many studies had high risk of bias scores on the QUADAS-2 assessment but low concerns for applicability. Sensitivity and specificity for CRP $\geq 20 \mathrm{mg} / \mathrm{L}$ (5 studies, 252 participants) was 59\% (95\% Cl 48-69) and 83\% ( $95 \%$ Cl 74-89) respectively. SROC curves are provided for each index test. At selected specificity of $80 \%$, the sensitivities for CRP (all cut-offs, 17 studies, 1404 participants), PCT ( all cut-offs, 6 studies, 231 participants) and IL6 (all cut-offs, 5 studies, 299 participants) were 59\%(95\% Cl 52-68), 56\%(95\% Cl 50-69) and 52\% (95\% Cl 50-86) respectively.

* Correspondence: angela.koech@aku.edu

'Department of Obstetrics and Gynaecology, Aga Khan University, P.O. Box 30270-00100, Nairobi, Kenya

Full list of author information is available at the end of the article

C The Author(s). 2020 Open Access This article is licensed under a Creative Commons Attribution 4.0 International License, which permits use, sharing, adaptation, distribution and reproduction in any medium or format, as long as you give appropriate credit to the original author(s) and the source, provide a link to the Creative Commons licence, and indicate if changes were made. The images or other third party material in this article are included in the article's Creative Commons licence, unless indicated otherwise in a credit line to the material. If material is not included in the article's Creative Commons licence and your intended use is not permitted by statutory regulation or exceeds the permitted use, you will need to obtain permission directly from the copyright holder. To view a copy of this licence, visit http://creativecommons.org/licenses/by/4.0/. The Creative Commons Public Domain Dedication waiver (http://creativecommons.org/publicdomain/zero/1.0/) applies to the data made available in this article, unless otherwise stated in a credit line to the data. 


\begin{abstract}
(Continued from previous page)
Conclusions: There is insufficient evidence to support use of CRP, procalcitonin or IL6 in maternal blood for diagnosis of HCA/Funisitis in PPROM. This review followed recommended methodology and data analytic methods that made the most of the data regardless of the different cut-offs used. However, the evidence is based on few studies with generally small sample sizes, poor-quality scores and substantial heterogeneity. There is a need for good-quality diagnostic accuracy studies to better assess the role of these biomarkers in PPROM.
\end{abstract}

Systematic review registration: PROSPERO registration number: CRD42015023899, registered on 8 October 2015.

Keywords: Inflammatory markers, Chorioamnionitis, C-reactive protein, Procalcitonin, Interleukin 6

\section{Background}

In preterm prelabour rupture of membranes (PPROM), the decision for delivery is a delicate balance that considers risks of preterm birth versus risks of infection from continuing pregnancy $[1,2]$. Typically, expectant management is carried out until the patient develops clinical signs suggestive of infection or until an appropriate gestation for safe delivery is reached. If clinical features of infection or inflammation are detected, then usually delivery is initiated. These clinical features can be thought of as an existing test. Inflammatory markers may form a suitable replacement test in place of the clinical features as the latter often become evident late or remain absent even in the presence of chorioamnionitis [3]. If inflammatory markers assayed in maternal blood are found to be sufficiently accurate in the diagnosis of chorioamnionitis, they can influence clinical decision-making and reduce reliance on clinical features alone. Early diagnosis of infection can advise therapeutic interventions such as delivery and antibiotic administration [4].

Maternal serum offers a readily accessible biological sample for assay of inflammatory markers and is preferred over alternative samples such as amniotic fluid which are harder to obtain [5] and not always available in non-specialist centres. Cord blood is an alternative sample, but its availability only after delivery precludes its use in decision-making during pregnancy.

There is no consensus on a suitable reference standard for diagnosis of chorioamnionitis [5-8]. We opted to use histologic chorioamnionitis (HCA) and/or funisitis as the reference standard for this review because standard criteria for ascertainment have existed for many years [9], its assessment is objective where these criteria are applied and there is good correlation with neonatal outcomes [10].

Several studies have evaluated maternal inflammatory markers for diagnosis of chorioamnionitis in PPROM with varying results and recommendations. Current guidelines $[1,4]$ do not recommend use of these markers alone for diagnosing infection in PPROM, but despite this, many clinicians continue to use these tests in PPROM with the results potentially influencing clinical decision-making [11]. Older reviews suggested CRP is useful in diagnosis of chorioamnionitis [12], but more recent systematic reviews [6-8] give no clear evidence for this recommendation. Prior systematic reviews have evaluated the role of C-reactive protein (CRP) in PPROM $[6,7]$ and do not recommend its use for predicting chorioamnionitis. However, these reviews were based on few studies [6-8], demonstrated marked heterogeneity $[6,7]$ and used data analysis methods that are not recommended $[6,8]$. Several studies assessing CRP and other inflammatory markers have since been published.

The objective of this review was to evaluate the accuracy of maternal blood inflammatory markers: C-reactive protein (CRP), procalcitonin (PCT) and interleukin 6 (IL6) in the diagnosis of histologic chorioamnionitis and/or funisitis in PPROM and to assess the sources of heterogeneity in estimates of diagnostic accuracy.

\section{Methods}

This systematic review of diagnostic accuracy employed methodological approaches recommended in the Cochrane Handbook for Systematic Reviews of Diagnostic Test Accuracy [13] and followed a prospectively prepared protocol [14] registered with PROSPERO CRD42015023899. This report complies with the Preferred Reporting Items for Systematic Reviews and Meta-analyses of Diagnostic Test Accuracy Studies, the PRISMA-DTA statement [15], and PRISMA-DTA checklists are provided as Additional file 1.

The inclusion criteria were studies of pregnant women with PPROM before 37 completed weeks of gestation. The tests of interest were CRP, PCT and IL6 performed on a maternal blood sample obtained prior to delivery, with any cut-off and any method of assay. The reference standard for chorioamnionitis was histologic chorioamnionitis and/or funisitis (HCA/Funisitis)-where a definition or diagnostic criteria was provided or a specification of histologic or microscopic assessment of the placenta was indicated or where the placenta was assessed by a pathologist. Any study design where the results of the index test were compared with the reference standard and reported data allowed extraction of 2 $\times 2$ data was eligible. 
We aimed to identify relevant studies published in peer-reviewed journals. We searched MEDLINE, EMBASE and The Cochrane Library from inception to 5 Jan 2020 and performed manual searches on reference lists of included articles and previous related reviews. The search strategy included a combination of subject headings and free-text terms related to the index test and target population only. We did not use any filters or search terms for the study design $[16,17]$ nor did we include the term 'diagnostic study'. There were no restrictions for language, publication dates or geographical setting in the electronic search. Where the database allowed, the limit for 'Humans' was applied. The search strategy is provided in Additional file 2.

Initial screening of titles and/or abstracts and subsequent in-depth review of full texts were done independently by 2 reviewers each (AKE, GO and AMM). Disagreements were resolved by consensus that included a third reviewer (AMM). Despite no restrictions for language in the electronic search and abstract screening, studies with non-English/non-French full texts were excluded due to anticipated difficulties in obtaining translations. Data extraction was done independently by 2 reviewers (AKE and GO) using a custom data extraction form that was piloted on 3 randomly selected eligible studies. Extracted fields included study characteristics (study design, setting, year of study, inclusion criteria, gestational age range), characteristics of the index tests (index test, method of assay, cut-off(s), timing of index test relative to delivery), clinical management of participants (antibiotic use, steroids, tocolysis) and indices of diagnostic accuracy. True positive, true negative, false positive and false negative values $(2 \times 2$ data) for each test in each study and for each cut-off reported were extracted or calculated from indices of diagnostic accuracy provided. In studies with a wide range of clinical diagnoses (e.g. including preterm labour with intact membranes) or wide gestational age range (e.g. including term PROM), $2 \times 2$ data was extracted for the PPROM subgroup where this was reported separately. Authors of otherwise eligible studies but with missing, unclear or conflicting $2 \times 2$ data were contacted by email.

A review-specific checklist derived from the Quality Assessment of Diagnostic Accuracy Studies 2 (QUADAS-2) [18] tool was used to assess the methodological quality of included studies. Assessments were done by 2 reviewers (GO and AKE) independently with disagreements resolved by consensus. Studies with a low risk of bias in patient selection were those that employed consecutive or random sampling and excluded women with clinical features of chorioamnionitis and/or preterm labour at the time of presentation with PPROM. Patient selection criteria that were potential sources of bias included selecting patients based on availability of other tests or completeness of records, restricting patients to a particular duration of PPROM and excluding women with common pregnancy-related or medical conditions. For the reference standard, objective and blinded assessment of the placenta was considered to have low risk of bias. The study flow and timing was considered to be of low risk of bias if the interval between blood sampling and delivery (proxy for placental assessment) was $\leq 72 \mathrm{~h}$ and if data were analysed and reported for $\geq 90 \%$ of included participants.

We obtained study estimates of sensitivity, specificity and corresponding 95\% confidence intervals (CI) and displayed these on coupled forest plots. Meta-analysis was carried out if the number of studies in each index test category was $\geq 3$. All reference standards were considered together as one. Summary receiver operator characteristic (SROC) curves were constructed for each test regardless of cut-off using the Rutter and Gatsonis' Hierarchical SROC (HSROC) model [19]. HSROC analysis was conducted using the NLMIXED procedure in $\mathrm{SAS}^{\circ}$ (University Edition 2016, Cary, NC), and the parameter estimates obtained were then inputted into Cochrane Review Manager (RevMan, version 5.3, Copenhagen) for construction of the curves [20]. The HSROC analysis is a random effects model, and it accounts for the correlation between sensitivity and specificity across the studies with changes in threshold [19, 20]. It makes the most use of the data as studies are pooled regardless of differences in cut-offs [20]. For studies using the same cut-off, we used bivariate analysis to obtain summary sensitivity and specificity and corresponding 95\% CIs. To aid understanding of the findings, we derived normalised frequencies assuming a patient population of 100 women and a prevalence obtained from the median prevalence of the included studies [21]. For SROC curves, we chose a false positive rate and derived corresponding sensitivity and confidence intervals from the model [21].

Heterogeneity assessment for studies using the same cut-off was carried out by visually inspecting the $95 \%$ prediction regions on SROC curves [20]. For the other studies, further exploration for causes of heterogeneity was carried out where the number of studies exceeded 5 and each subgroup had at least 2 studies. We aimed to evaluate the following as possible sources: assay type, pre-specified cut-off, interval between sampling and delivery and the risk of bias score in the patient selection domain of the QUADAS-2. These characteristics were added as binary covariates to the HSROC models in SAS $^{\bullet}$ (University Edition 2016, Cary, NC). Pairs of SROC curves were constructed by inputting the parameters into Cochrane Review Manager (RevMan, version 5.3, Copenhagen). For simplicity, the shape parameter was 
assumed to be the same in the 2 subgroups. Chi-squared test was used to compare the 2-Log likelihoods to test for differences in SROC curves between subgroups. Covariates were applied to the model one at time and curves compared for each characteristic in turn. We did not construct models with more than one covariate due to limited power in the setting of few studies [20,22].

We performed sensitivity analysis to investigate the possible influence of including studies with a narrower gestational age range (limiting the review to studies with gestational age above 24 weeks), year of publication (limiting the review to studies published after year 2000) and limiting the review to studies with low concerns for applicability on the patient selection domain of the QUADAS-2 assessment. Pairs of SROC plots were constructed and comparison done visually [20]. No assessment of publication bias was performed as included studies were few or too heterogeneous [23, 24].

\section{Results}

\section{Results of the search}

The search yielded 3020 unique records of which 25 (25 publications, 23 unique studies) were included (Fig. 1). Twenty-one of the 46 potentially eligible studies were excluded due to missing or unclear $2 \times 2$ data. More information on these studies is provided in Additional file 3. No additional data was obtained from contacted authors.

\section{Characteristics of included studies}

The studies were published between 1983 and 2019 and conducted in 13 countries. Twenty studies were prospective cohort design and 3 studies retrospective cohort design. All were conducted in hospital inpatient settings with majority at teaching/university hospitals. In total, there were 1717 participants, 902 of whom had HCA/ funisitis; median prevalence 50\%; and inter-quartile range $38 \%$ to $57 \%$. Characteristics of included studies are summarised in Table 1.

All studies reported data for preterm gestation $(<37$ weeks) at the time of prelabour rupture of membranes (PROM), but the specific gestational age range for eligibility varied greatly among the included studies. Methods used to establish gestational age were unreported in most studies [26, 28-31, 34-36, 39, 41, 43, 45] except for $5[27,32,40,44,49]$ which used a combination of last menstrual period and ultrasound. Where reported, diagnosis of PROM was made by clinical assessment (speculum examination) with some studies $[27,28,31,33,35,38,40,43-45,49]$ conducting further confirmatory testing on all or some of the patients. Management of PPROM was largely expectant with monitoring of fetal well-being, surveillance for clinical features of chorioamnionitis and monitoring for signs of labour. Use of antibiotics, steroids and/or tocolytics where reported was universal or selective-dependent on gestational age or clinical features. Reasons for delivery included gestational age greater than 34 weeks [36, $40,45]$, failed tocolysis or refractory labour [26-28, 35], completion of steroids or confirmed pulmonary maturity [26, 27, 44], foetal distress/abnormal cardiotocogram [26, 27, 35, 36, 44], suspected abruption [35] and/or other obstetric complications [36, 40,44]. Six studies specified that clinical features of chorioamnionitis were an indication for delivery $[26,28,29,36,44,49]$. According to the definitions of reference standard provided, 11 studies [26, 28,31,35, 36, 40-44, 50] reported the index test against a reference standard of HCA and/ or funisitis, $10[27,29,30,32,34,39,45,46,48,49]$ reported HCA alone and 2 studies [33, 38] reported funisitis alone. Characteristics of included studies are outlined in Table 1. Studies evaluated the index tests over a wide range of cut-offs. More characteristics of index tests are provided in Additional file 4.

\section{Methodological quality of included studies}

Many studies were poorly reported, and 22 out of 23 were found to be at high risk of bias in at least 1 of the 4 domains of the QUADAS-2 (QUADAS-2 whiting) tool (Fig. 2, Additional file 5). In the 'Patient selection' domain, we judged 14 of the 23 studies to be at high risk of bias largely due to inappropriate exclusions such as excluding women based on duration after PPROM [35, 38 , not explicitly excluding women with clinical features of chorioamnionitis at the time of PPROM or at the time of admission [26, 27, 31, 34, 38, 40], basing exclusions on availability or ability to perform other tests [31, $40,45]$, excluding women due to missing data [34, 35, 50] and excluding women with common conditions and complications of pregnancy that often coexist with PPROM [32, 36, 39, 40]. In the 'Index test' domain, all tests were considered to be 'blinded' because maternal blood was collected before delivery and assessed on automated assays. Studies where the cut-offs used were not pre-specified [29, 31, 32, 35, 39, 43, 45, 49] but determined from the study data were also deemed to be at high risk of bias. Only 6 studies [27, 29, 33, 38, 40, 46] explicitly reported blinding in placental assessment. There were marked differences in the timing of collection of maternal blood, and many studies failed to report this clearly [26, 34, 36, 40]. We assumed $a \leq 72$-h interval between maternal blood sampling and delivery to be appropriate as we felt the relationship between the index test and the outcome at placental assessment would be preserved. Only 11 studies [28-31, 33, 35, 36, 38, 41, 42, 49] had samples drawn within this interval. Studies that used samples obtained close to the time of admission or the time of PPROM would be at higher risk of bias due 


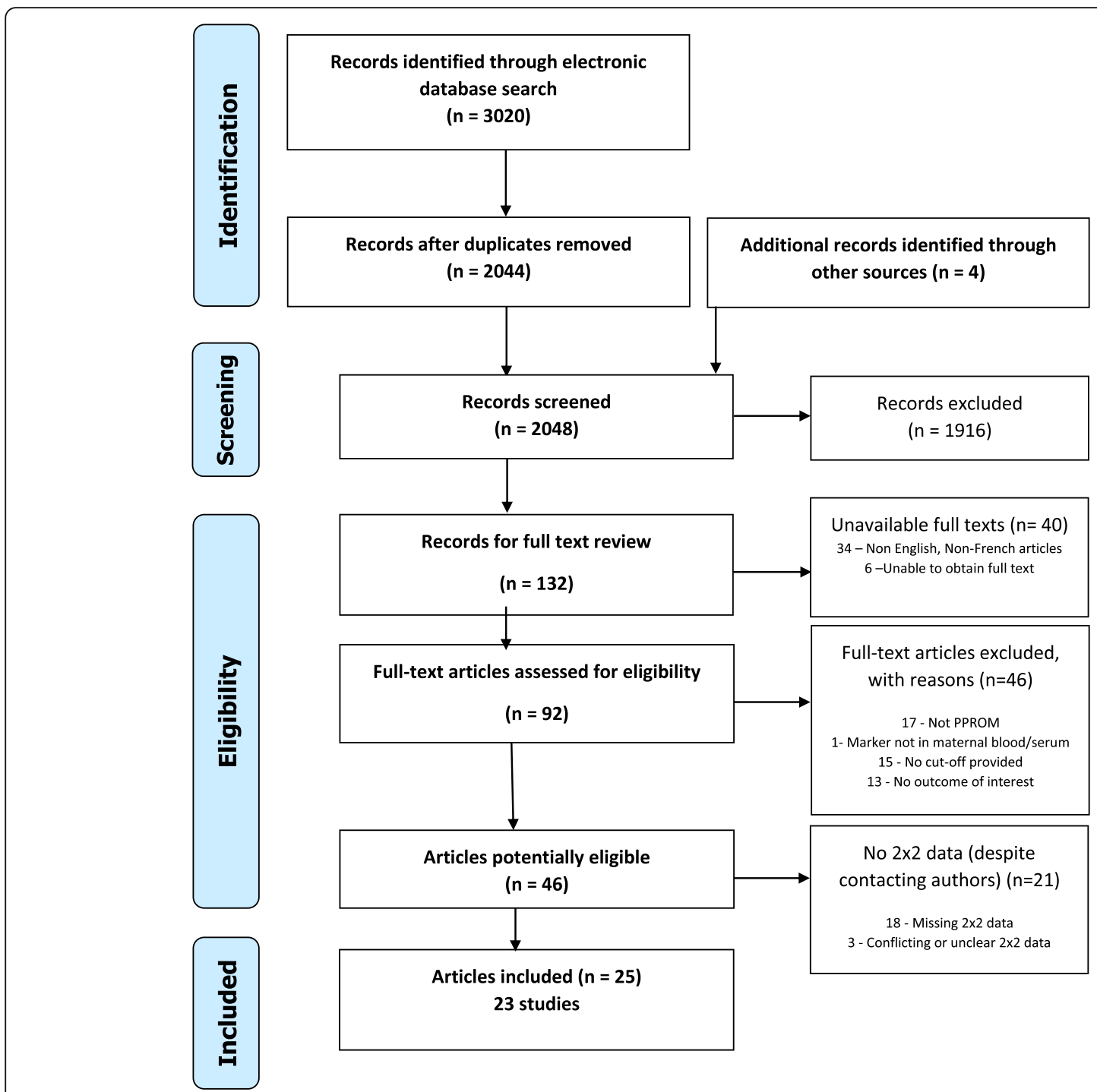

Fig. 1 Study flow diagram. PPROM, preterm prelabour rupture of membranes. HCA, histologic chorioamnionitis. Figure modified from the PRISMA statement [25]

to variable lengths of latency after PPROM. All included studies had low concerns for applicability with regard to the index test and reference standard. In the 'Patient selection' domain, 5 studies $[26,27,31,35,38]$ were judged to have high concerns for applicability as they did not explicitly report exclusion of contractions or advanced cervical dilatation (preterm labour).

\section{Findings}

Seventeen studies evaluated CRP as the index test, 6 evaluated the role of PCT and 5 evaluated IL6. Sensitivity and specificity pairs and their confidence intervals are demonstrated in Fig. 3. The forest plot shows wide variability in the sensitivity and specificity for each index test group. Studies reported data against a wide range of index test cut-offs (Fig. 3). Figures 4 and 5 show the various studies each plotted in ROC space as a single sensitivity-specificity point. The sizes of the individual points reflect the study sample size, and the scatter gives an impression of the heterogeneity in the findings. For CRP, 5 studies reported findings at a cutoff of $20 \mathrm{mg} / \mathrm{L}$. A summary point of sensitivity and specificity is provided for this test group, and the large 95\% prediction region reflects substantial heterogeneity. For the other test groups, a SROC curve is plotted for the range of sensitivity and specificity from the included studies. The closer the curve to the top left corner, the better the overall accuracy. The wide scatter of the study points in these plots suggests substantial heterogeneity. 


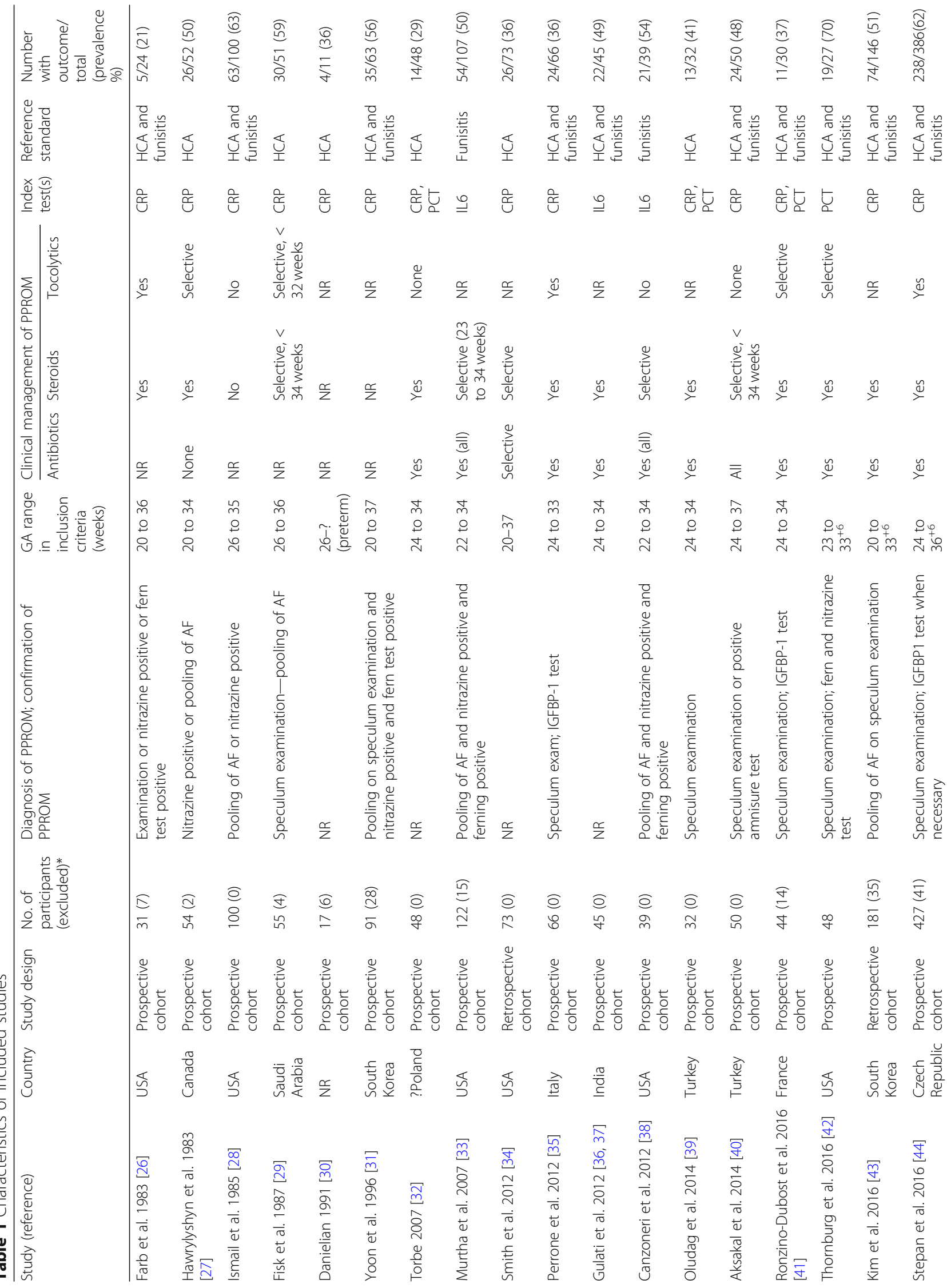




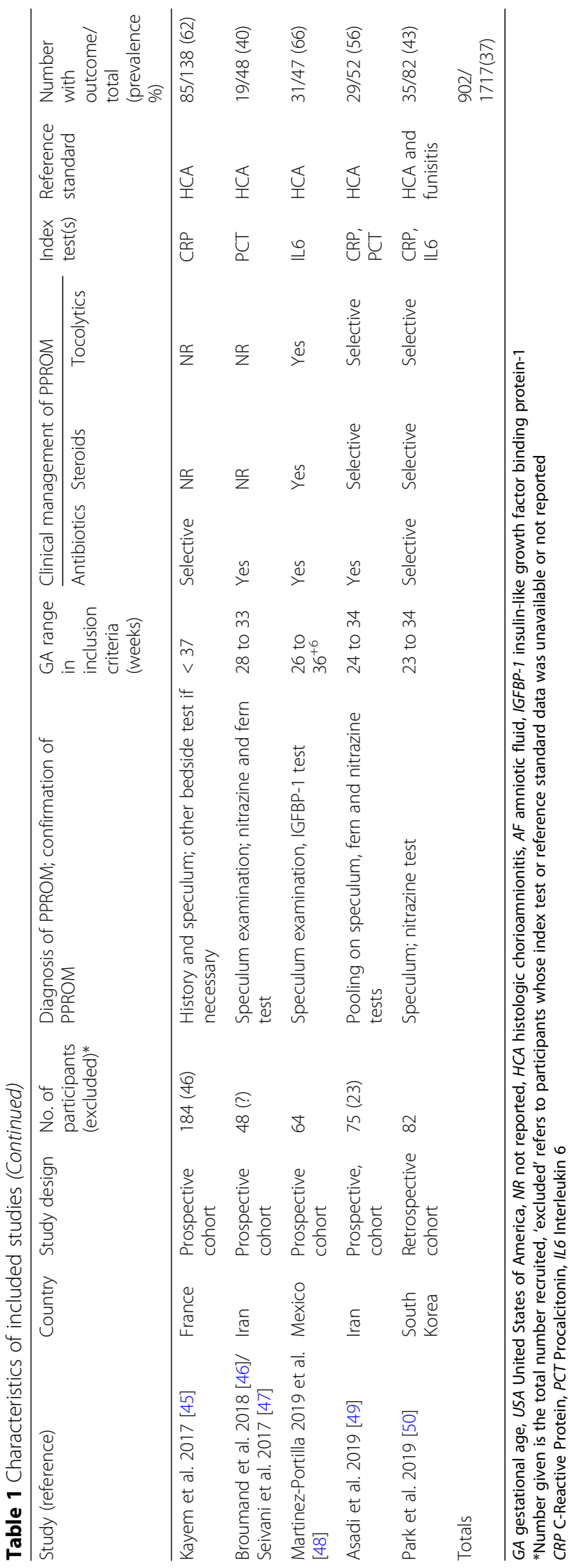




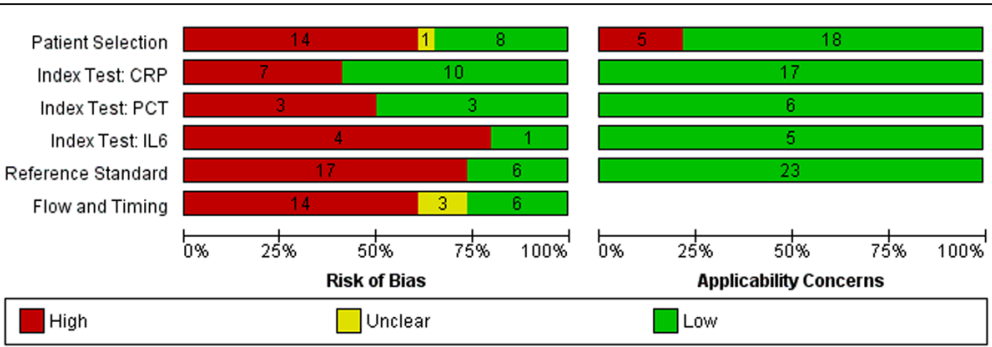

Fig. 2 Risk of bias and applicability concerns graph [18] for included studies. CRP, C-reactive protein; PCT, procalcitonin; IL6, interleukin 6

\section{Findings of heterogeneity assessments}

There was some heterogeneity as demonstrated by the 95\% prediction region on the SROC (Fig. 4) for the studies reporting CRP at $20 \mathrm{mg} / \mathrm{L}$. Further heterogeneity assessments revealed likely sources as interval between maternal blood sampling and delivery, nature of index test cut-off (predetermined or not), risk of bias score in the patient selection domain and assay type (Table 2, Additional file 6).

\section{Findings of sensitivity analysis}

Sensitivity analysis for CRP were performed to assess the influence of including studies based on gestational age range, applicability concerns in the patient selection domain and year of publication. Year of publication was not assessed for PCT and IL6 as all studies were published after the year 2000. All IL6 studies had low applicability concerns in the patient selection domain, so this was not assessed. Results of the sensitivity analysis are given in Table 2 and Additional file 7.

Findings of this diagnostic review are summarised in the summary of findings table, Table 3.

\section{Discussion \\ Main findings}

The results of this review show the 3 tests have high false positive rates (low specificity) and high false negative rates (low sensitivity) in the diagnosis of histologic chorioamnionitis and/or funisitis (see Summary of findings table-interpretation). These findings

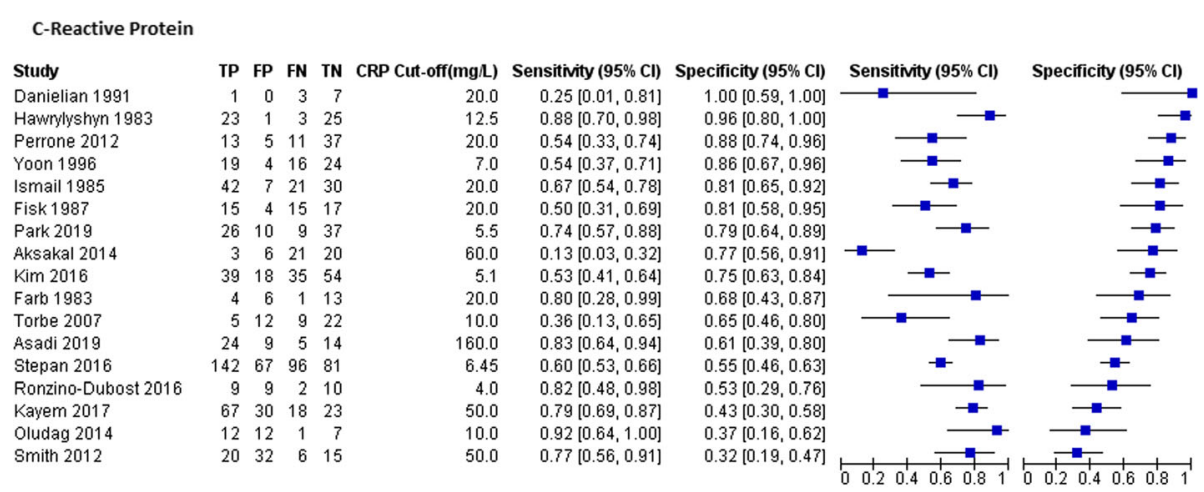

Procalcitonin



Fig. 3 Forest plot showing sensitivity and specificity for included studies. TP—true positive, FP—false positive, FN-false negative, TN—-true negative, $\mathrm{Cl}$-confidence interval, CRP_C-reactive protein, PCT—procalcitonin, IL6-interleukin 6. Studies are ordered by specificity in descending order for each index test group 

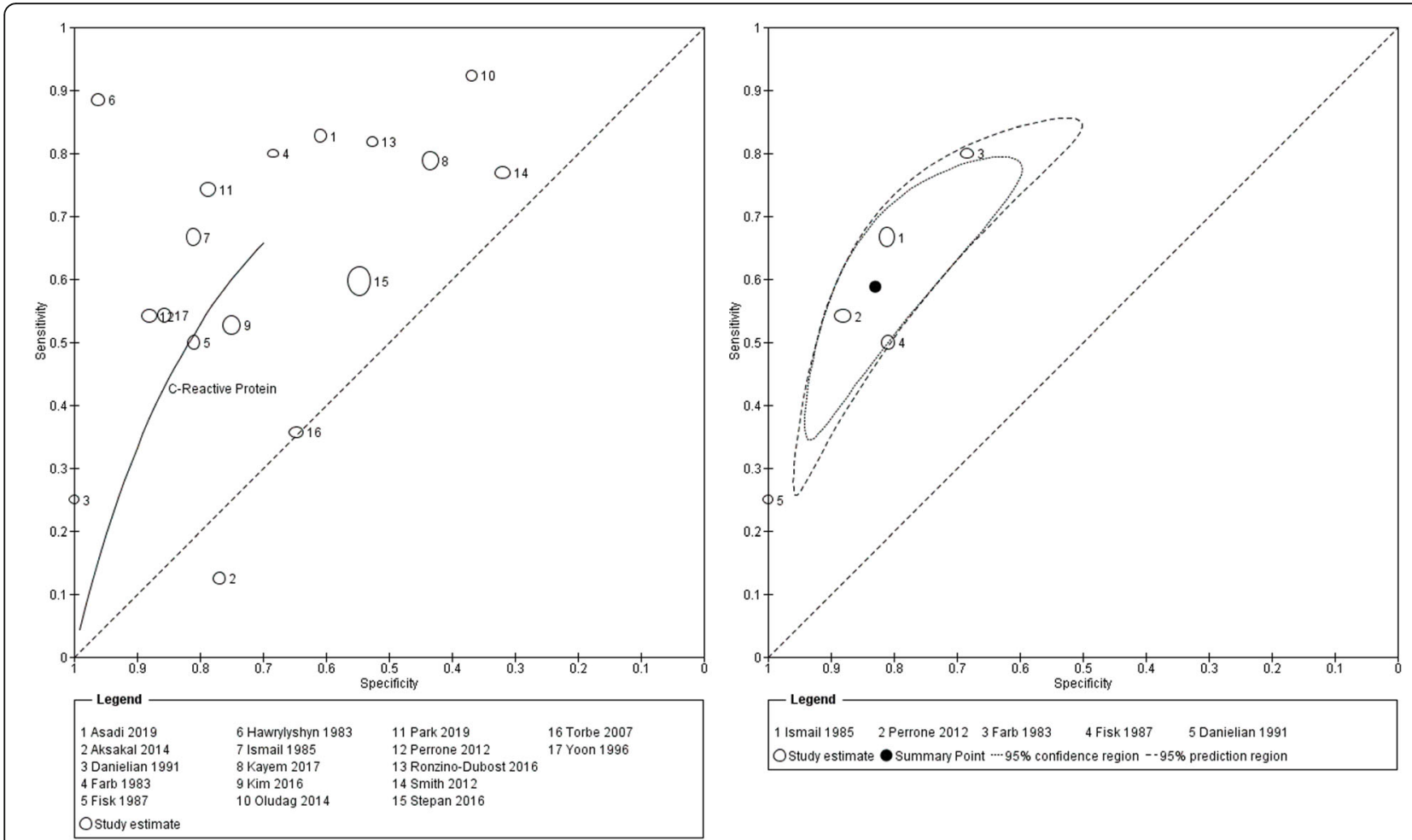

Fig. 4 Summary ROC curve: C-reactive protein for histologic chorioamnionitis and/or funisitis; Curve 1 - C-reactive protein all studies. Curve 2 - Creactive protein at $20 \mathrm{mg} / \mathrm{L}$ cutoff
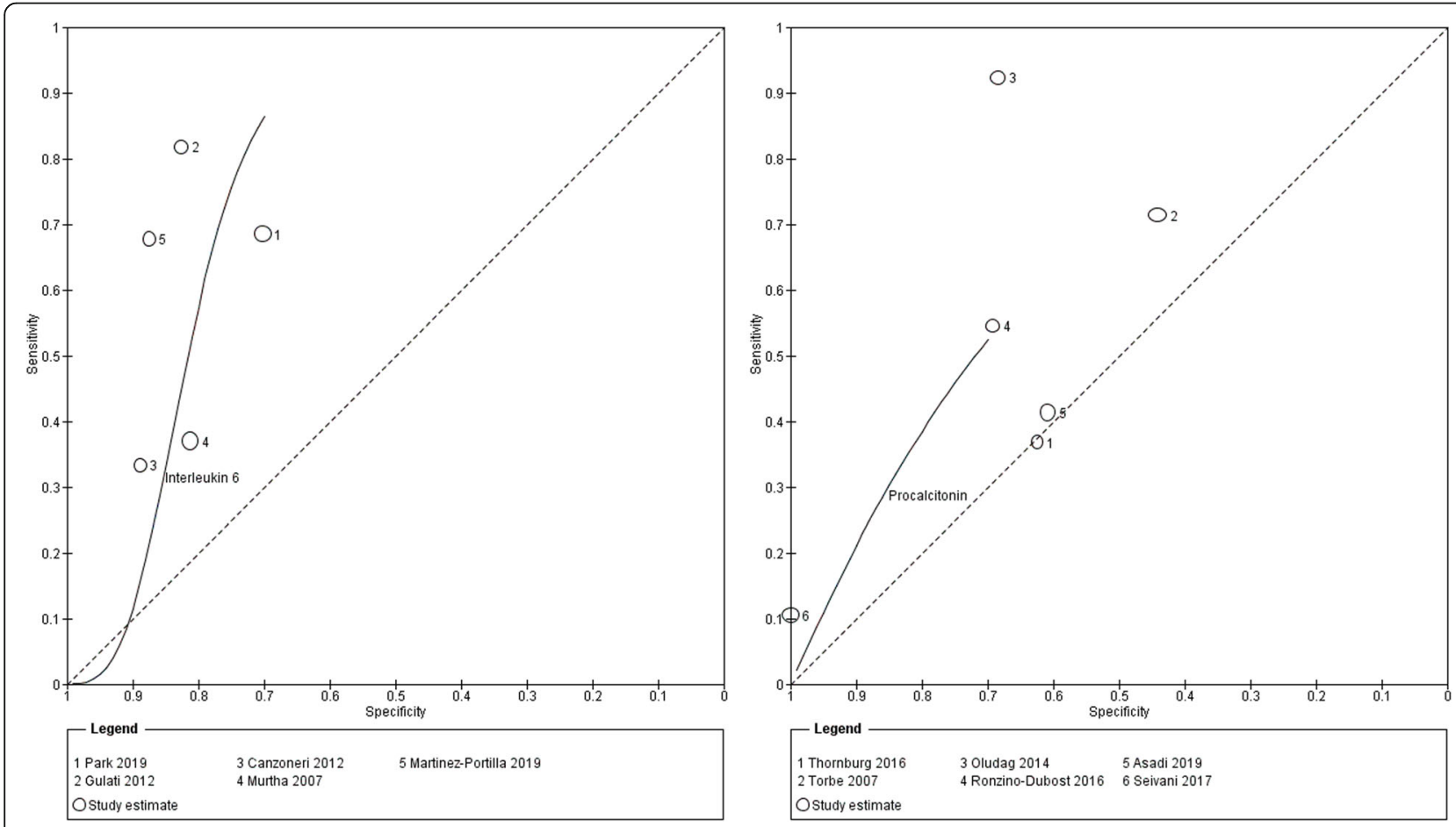

Fig. 5 Summary ROC curves: interleukin 6 and procalcitonin for histologic chorioamnionitis and/or funisitis 
Table 2 Heterogeneity assessments and sensitivity analysis

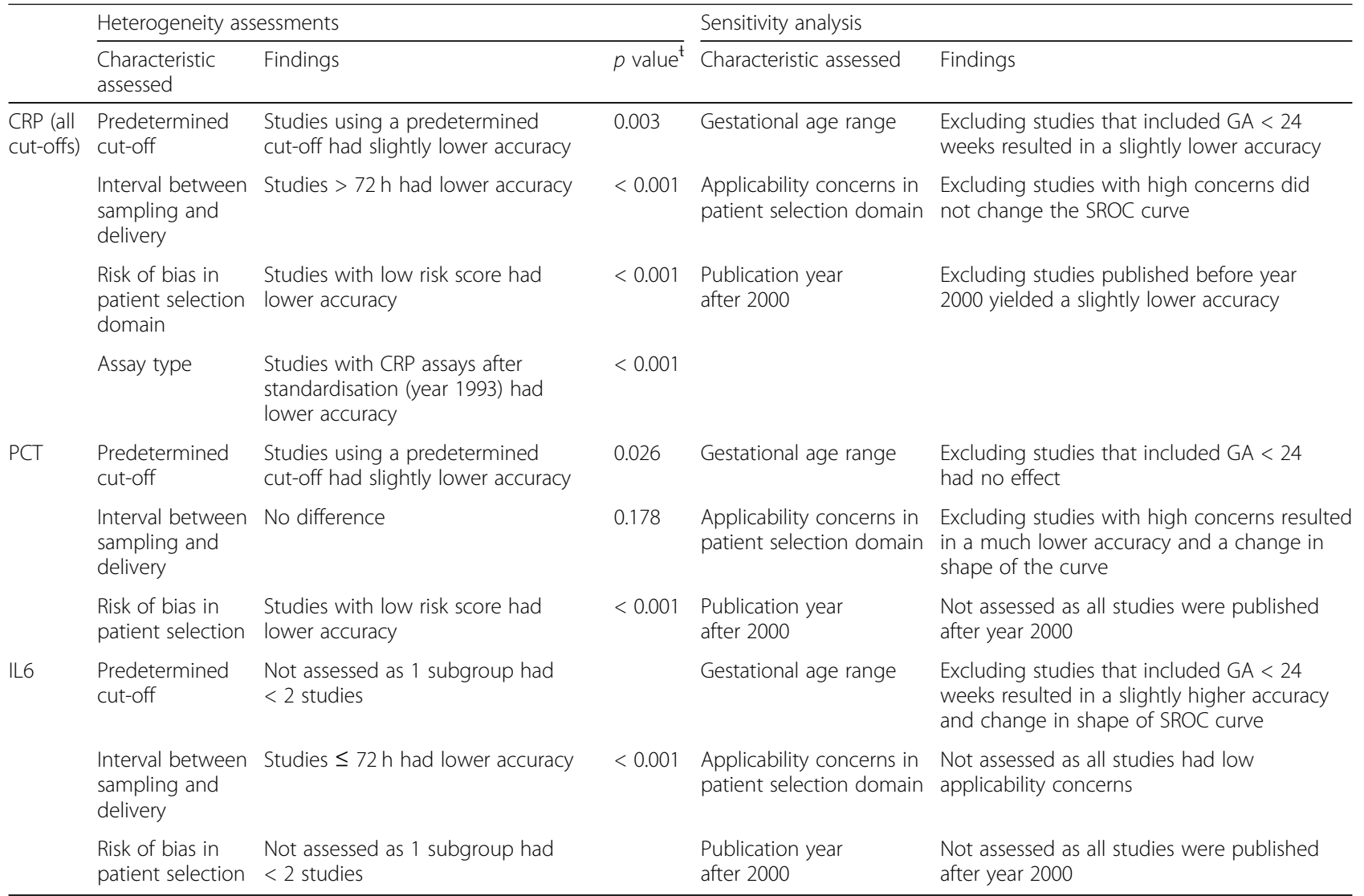

More information is provided in Additional files 6 and 7

t'Likelihood ratio test

CRP C-Reactive Protein, PCT Procalcitonin, SROC Summary Receiver Operating Characteristic, IL6 Interleukin 6

are obtained in the background of few included studies with generally small sample sizes, poor quality assessments and substantial heterogeneity.

\section{Strengths and limitations}

The findings of this review need to be evaluated with the knowledge of various strengths and weaknesses both of the included studies and those of the review methods. Included studies were few in number and generally had small sample sizes. This affects the precision and applicability of the findings, especially in the face of substantial heterogeneity. Studies were of poor quality with a high risk of bias in 1 or more domains. Poor reporting limited the assessment of methodological quality and applicability of many of the included studies. Findings of these studies are likely to be affected by various biases due to poor study design.

We have conducted this review following recommendations of the Cochrane group of diagnostic reviews [20] and following a prospectively registered protocol [14]. We employed a broad search strategy with search terms that did not include the reference standard and did not use a filter for 'diagnostic studies' [51]. However, a large proportion of potentially eligible studies were excluded due to inability to extract $2 \times 2$ data. Despite contacting authors of these studies, no additional data were obtained. We only included studies published in English and French and failed to obtain full texts of 6 articles. Our review was also limited to published studies only, limiting its representativeness.

Our review question limited the studies to those addressing a specific clinical condition in pregnancy, PPROM. This reduced chances of pooling together test accuracy indices that are different due to differences in patient characteristics and probability of disease [52]. All included studies had low concerns for applicability in the index test and reference standard domains. High applicability concerns arose in the patient selection domain particularly due to failure to explicitly exclude patients with preterm labour and perhaps due to poor reporting of inclusion criteria in some studies. We explored potential sources of heterogeneity where possible, but some subgroup analysis could not be carried out due to the few studies. We assumed the same shape (parallel curves) in comparing SROCs of subgroups due to the small number of 
Table 3 Summary of findings table

\begin{tabular}{|c|c|c|c|c|c|c|c|c|c|}
\hline \multicolumn{10}{|c|}{$\begin{array}{l}\text { Maternal inflammatory markers for chorioamnionitis in preterm prelabour rupture of membranes(PPROM): a systematic review and meta-analysis of } \\
\text { diagnostic test accuracy studies }\end{array}$} \\
\hline Question & \multicolumn{9}{|c|}{ In pregnant women with PPROM, can maternal serum inflammatory markers be used to diagnose chorioamnionitis? } \\
\hline Population & \multicolumn{9}{|c|}{ Pregnant women with PPROM } \\
\hline Studies & \multicolumn{9}{|c|}{ Any study design where the index test is compared against the reference standard } \\
\hline Index test & \multicolumn{9}{|c|}{ C-reactive protein (CRP), procalcitonin (PCT) and interleukin 6 (IL6) assessed in maternal serum before delivery } \\
\hline $\begin{array}{l}\text { Reference } \\
\text { standard }\end{array}$ & \multicolumn{9}{|c|}{ Histologic chorioamnionitis (HCA) and/ or funisitis } \\
\hline $\begin{array}{l}\text { Prevalence } \\
\text { of disease }\end{array}$ & \multicolumn{9}{|c|}{$\begin{array}{l}\text { Median prevalence } 50 \% \text { (range } 21-70 \% \text {, IQR } 38 \text { to } 57 \% \text { ) } \\
23 \text { studies with a total of } 1717 \text { pregnant women with PPROM, } 902 \text { of whom had HCA/funisitis }\end{array}$} \\
\hline Quality & \multicolumn{9}{|c|}{$\begin{array}{l}\text { Included studies were generally of poor quality with all studies at high risk of bias in at least one domain (QUADAS-2). There were few } \\
\text { studies with high applicability concerns and only in the patient selection domain. }\end{array}$} \\
\hline \multirow[t]{2}{*}{ Index test } & \multirow[t]{2}{*}{$\begin{array}{l}\text { Studies } \\
\text { (participants) }\end{array}$} & \multirow[t]{2}{*}{$\begin{array}{l}\text { Sensitivity } \\
(95 \% \text { Cl) }\end{array}$} & \multirow[t]{2}{*}{$\begin{array}{l}\text { Specificity } \\
(95 \% \mathrm{Cl})\end{array}$} & \multirow[t]{2}{*}{ Heterogeneity } & \multirow[t]{2}{*}{$\begin{array}{l}\text { Sensitivity } \\
\text { analysis }\end{array}$} & \multicolumn{4}{|c|}{$\begin{array}{l}\text { Interpretation: assuming a patient population of } \\
100 \text { pregnant women with PPROM and } \\
\text { prevalence of 50\%* }\end{array}$} \\
\hline & & & & & & $\begin{array}{l}\text { Correctly } \\
\text { diagnosed } \\
\text { cases (TP) }\end{array}$ & $\begin{array}{l}\text { Missed } \\
\text { cases } \\
\text { (FN) }\end{array}$ & $\begin{array}{l}\text { Unnecessary } \\
\text { interventions } \\
\text { (FP) }\end{array}$ & $\begin{array}{l}\text { True } \\
\text { reassurance } \\
\text { of no } \\
\text { disease (TN) }\end{array}$ \\
\hline $\begin{array}{l}\text { CRP at } \\
20 \mathrm{mg} / \mathrm{L}^{+}\end{array}$ & $5(252)$ & $\begin{array}{l}59 \% \\
(47.7-69.0)\end{array}$ & $\begin{array}{l}83 \% \\
(74.0-89.2)\end{array}$ & $\begin{array}{l}\text { High heterogeneity } \\
\text { despite common cut-off }\end{array}$ & & 30 & 21 & 9 & 42 \\
\hline $\begin{array}{l}\text { CRP at all } \\
\text { cut-offs }^{\ddagger}\end{array}$ & $17(1404)$ & $\begin{array}{l}59 \% \\
(52.0-67.6)\end{array}$ & $80 \%$ & $\begin{array}{l}\text { Partially explained by } \\
\text { nature of cut-off used, } \\
\text { sampling interval, risk of } \\
\text { bias in the patient selec- } \\
\text { tion domain and type of } \\
\text { CRP assay }\end{array}$ & $\begin{array}{l}\text { Sensitive to } \\
\text { gestational age } \\
\text { range for study } \\
\text { inclusion and } \\
\text { year of } \\
\text { publication }\end{array}$ & 30 & 20 & 10 & 40 \\
\hline $\begin{array}{l}\text { PCT at all } \\
\text { cut-offs }^{\ddagger}\end{array}$ & $6(231)$ & $\begin{array}{l}56 \% \\
(49.9-68.9)\end{array}$ & $80 \%$ & $\begin{array}{l}\text { Partially explained by } \\
\text { nature of cut-off used } \\
\text { and risk of bias in the pa- } \\
\text { tient selection domain of } \\
\text { QUADAS-2 }\end{array}$ & $\begin{array}{l}\text { Sensitive to } \\
\text { applicability } \\
\text { concerns score } \\
\text { in the patient } \\
\text { selection domain } \\
\text { of QUADAS-2 }\end{array}$ & 28 & 22 & 10 & 40 \\
\hline $\begin{array}{l}\text { IL6 at all } \\
\text { cut-offs }\end{array}$ & $5(299)$ & $\begin{array}{l}52 \% \\
(50.0-85.8)\end{array}$ & $80 \%$ & $\begin{array}{l}\text { Partially explained by } \\
\text { sampling interval }\end{array}$ & $\begin{array}{l}\text { Sensitive to } \\
\text { gestational age } \\
\text { range for study }\end{array}$ & 26 & 24 & 10 & 40 \\
\hline
\end{tabular}

The results on this table should not be interpreted in isolation from the results in the main body of the text of the review

*Median prevalence from included studies

${ }^{\dagger}$ Estimate from the summary point from bivariate analysis

\#Sensitivity derived from HSROC analysis assuming a specificity of $80 \%$ (false positive rate of $20 \%$ )

CRP C-Reactive Protein, PCT Procalcitonin, IL6 Interleukin 6

studies-this would miss situations where the accuracy of the test varied with threshold in a different manner in the 2 subgroups compared.

Previous reviews $[6,7]$ examining the role of inflammatory markers in diagnosis of chorioamnionitis in PPROM had few studies, high between-study heterogeneity and differences in cut-offs that prevented pooled analysis. We identified more studies through our broader search criteria. These reviews $[6,7]$ also used methods of analysis that are no longer recommended. We used HSROC analysis [19, 20], a method that allowed pooling of studies with different cut-offs hence making efficient use of the data and maximising power [20]. We also assessed heterogeneity and identified likely sources. Despite these differences, our findings are in agreement with previous reviews that there is no evidence to support use of CRP, PCT or IL6 in the diagnosis of chorioamnionitis.

\section{Conclusions \\ Implications for clinical practice}

The proposed clinical role of the tests in PPROM is to guide interventions such as delivery or expectant management by appropriately identifying which pregnancies have chorioamnionitis. We have found insufficient evidence to recommend the use of either CRP, PCT or IL6 in maternal blood as a solitary test for the diagnosis of HCA/Funisitis in PPROM. Though it is relatively easy to obtain maternal blood for laboratory evaluation of these markers, the high false 
positive rates mean the tests should not be relied upon for important clinical decisions such as delivery. False positive results would have greater negative implications as they would result in iatrogenic preterm delivery with no indication. False positives at earlier gestations greatly could significantly impact neonatal outcome and survival.

Whether use of these tests should be recommended also depends on existence of and diagnostic performance of alternative tests in similar roles. Inflammatory markers in amniotic fluid may have better diagnostic performance than tests in maternal blood [53] but are limited by the complexity of amniotic fluid collection, increased costs and lower acceptability to women. Alternative approaches may be to combine these tests with other laboratory and clinical markers or to conduct serial tests [4]. This review did not examine these alternative tests and approaches.

\section{Implications for research}

This review has demonstrated several weaknesses in the included studies and significant heterogeneity in findings that limit our ability to make reliable conclusions. There is need for better designed diagnostic accuracy studies where an effort is placed to reduce the various sources of bias as outlined in our quality assessments. In addition to assessing the role of the inflammatory marker, the contribution of other clinical and laboratory factors could be assessed jointly by regression modelling.

Several studies included in this report were poorly reported. Use of the standards for Reporting of Diagnostic Accuracy_STARD [54] — could reduce this and enable reviewers to correctly assess quality of studies and make more data available for review and meta-analysis.

\section{Supplementary information}

Supplementary information accompanies this paper at https://doi.org/10. 1186/s13643-020-01389-4.

Additional file 1:. Format: .docx Title "PRISMA-DTA Checklists" Completed PRISMA-DTA checklist for the systematic review.

Additional file 2:. Format: docx Title "Search strategy" - Table showing the search strategy for the review, Medline database on Ovid platform.

Additional file 3:. Format: .docx Title "Characteristics of Excluded Studies" - Table showing characteristics of studies excluded from the review due to missing or conflicting $2 \times 2$ data 1

Additional file 4:. Format: .docx Title "Characteristics of Index Tests in included studies" - Table showing the characteristics of all index tests in the included studies

Additional file 5:. Format: .png Title "Risk of Bias and Applicability Concerns Summary"

Additional file 6:. Format: .docx Title "Heterogeneity Assessments" Figures and text showing and describing findings of the heterogeneity assessments

Additional file 7:. Format: .docx Title "Sensitivity Analysis" - Figures showing sensitivity analysis for studies evaluating C- reactive protein.

\section{Abbreviations}

Cl: Confidence interval; CRP: C-reactive protein; FN: False negative; FP: False positive; HCA: Histological chorioamnionitis; HCA/Funisitis: Histologic chorioamnionitis and/or funisitis; HSROC: Hierarchical summary receiver operating characteristic; IL6: Interleukin 6; IQR: Inter-quartile range; NR: Not reported; PCT: Procalcitonin; PRISMA: Preferred Reporting Items for Systematic Reviews and Meta-Analyses; PRISMA-DTA: Preferred Reporting Items for Systematic Reviews and Meta-Analyses for Diagnostic Test Accuracy Studies; PPROM: Preterm prelabour rupture of membranes; PROM: Prelabour rupture of membranes; PROSPERO: International Prospective Register of Systematic Reviews; QUADAS-2: Quality Assessment of Diagnostic Accuracy Studies-2; ROC: Receiver operating characteristic; SROC: Summary receiver operating characteristic; STARD: Standards for Reporting Diagnostic Accuracy Studies; TN: True negative; TP: True positive

\section{Acknowledgements}

The authors would like to thank William Stones and Stella Glasmacher (University of St Andrews) for their advice on the design of the study and data analysis, Nasra Gathoni (Aga Khan University Library) for assistance with the electronic search strategy and retrieving full texts and Alex Maina and Anthony Etyang (KEMRI-Wellcome Trust Research Programme-Kilifi), Laura Hammitt (Johns Hopkins School of Public Health) for assistance in retrieving full texts and Kevin Juma for assistance with data management.

\section{Authors' contributions}

AKE, GO, AMM and MT developed the concept and design of the study and interpretation of data. AKE, GO and AMM performed data acquisition. AKE performed the electronic search and data analysis. All authors were involved in drafting and revising the article and final approval of this version and agree to be accountable for all aspects of the work.

\section{Funding}

This study was funded by Aga Khan University, postgraduate medical education seed fund, ref 2015/REC-33.

\section{Availability of data and materials}

The datasets used and analysed during the current study are available from the corresponding author on reasonable request.

Ethics approval and consent to participate

This study was exempted from ethical review.

Consent for publication

Not applicable

\section{Competing interests}

None of the authors have any competing interests to declare.

\section{Author details}

${ }^{1}$ Department of Obstetrics and Gynaecology, Aga Khan University, P.O. Box 30270-00100, Nairobi, Kenya. ${ }^{2}$ Department of Pathology, Aga Khan University, P.O. Box 30270-00100, Nairobi, Kenya.

Received: 13 January 2019 Accepted: 18 May 2020

Published online: 12 June 2020

\section{References}

1. Carroll SGM. Preterm prelabour rupture of membranes, Green-top Guideline No.44. Royal College of Obstetricians and Gynaecologists. 2010.

2. Acog. Practice bulletins No. 139: premature rupture of membranes. Obstet Gynecol. 2013;122(4):918-30.

3. Curtin WM, Katzman PJ, Florescue H, Metlay LA. Accuracy of signs of clinical chorioamnionitis in the term parturient. J Perinatol: official journal of the California Perinatal Association. 2013;33(6):422-8.

4. NICE. NICE guideline [NG25], Preterm labour and birth. National Institute for Health and Care Excellence; 2015.

5. Ohlsson A, Wang E. An analysis of antenatal tests to detect infection in preterm premature rupture of the membranes. Am J Obstet Gynecol. 1990; 162(3):809-18.

6. Trochez-Martinez RD, Smith P, Lamont RF. Use of C-reactive protein as a predictor of chorioamnionitis in preterm prelabour rupture of membranes: a 
systematic review. BJOG: An International Journal of Obstetrics and Gynaecology. 2007;114(7):796-801.

7. van de Laar R, van der Ham DP, Oei SG, Willekes C, Weiner CP, Mol BWJ. Accuracy of $\mathrm{C}$-reactive protein determination in predicting chorioamnionitis and neonatal infection in pregnant women with premature rupture of membranes: a systematic review. Eur J Obstet Gynecol Reprod Biol. 2009; 147(2):124-9.

8. Wiwanitkit V. Maternal C-reactive protein for detection of chorioamnionitis: an appraisal. Infect Dis Obstet Gynecol. 2005;13(3):179-81.

9. Redline RW, Faye-Petersen O, Heller D, Qureshi F, Savell V, Vogler C. Amniotic infection syndrome: nosology and reproducibility of placental reaction patterns. Pediatr Dev Pathol. 2003;6(5):435-48.

10. Smulian JC, Shen-Schwarz S, Vintzileos AM, Lake MF, Ananth CV. Clinical chorioamnionitis and histologic placental inflammation. Obstet Gynecol. 1999:94(6):1000-5.

11. Greenberg MB, Anderson BL, Schulkin J, Norton ME, Aziz N. A first look at chorioamnionitis management practice variation among US obstetricians. Infect Dis Obstet Gynecol. 2012;2012.

12. Bek KM, Nielsen FR, Qvist I, Rasmussen PE, Tobiassen M. C-reactive protein (CRP) and pregnancy. An early indicator of chorioamnionitis. A review. Eur J Obstet Gynecol Reprod Biol. 1990;35(1):29-33.

13. Deeks J, Bossuyt PM, Gatsonis C. Cochrane Handbook for Systematic Reviews of Diagnostic Test Accuracy Version 1.0: The Cochrane Collaboration; 2010.

14. Koech A, Mukaindo M, Omuse G, Temmerman M. Maternal inflammatory markers in the diagnosis of chorioamnionitis and prediction of neonatal sepsis in preterm pre-labour rupture of membranes: a systematic review. PROSPERO. 2015(CRD42015023899)

15. McInnes MDF, Moher D, Thombs BD, McGrath TA, Bossuyt PM, Clifford T, et al. Preferred reporting items for a systematic review and meta-analysis of diagnostic test accuracy studies. Jama. 2018;319(4).

16. Leeflang MMG, Scholten RJPM, Rutjes AWS, Reitsma JB, Bossuyt PMM. Use of methodological search filters to identify diagnostic accuracy studies can lead to the omission of relevant studies. J Clin Epidemiol. 2006;59(3):234-40.

17. Beynon R, Leeflang MMG, McDonald S, Eisinga A, Mitchell RL, Whiting $P$, et al. Search strategies to identify diagnostic accuracy studies in MEDLINE and EMBASE. The Cochrane database of systematic reviews. 2013;9: MR000022-MR.

18. Whiting PF, Rutjes AWS, Westwood ME, Mallet S, Deeks JJ, Reitsma JB, et al. QUADAS-2: a revised tool for the quality assessment of diagnostic accuracy studies. Ann Intern Med. 2011;155(4):529-36.

19. Rutter CM, Gatsonis CA. A hierarchical regression approach to meta-analysis of diagnostic test accuracy evaluations. Stat Med. 2001;20(19):2865-84.

20. Macaskill P, Gatsonis C, Deeks J, Harbord R, Takwoingi Y. Chapter 10 analysing and presenting results. Cochrane Handbook for Systematic Reviews of Diagnostic Test Accuracy The Cochrane Collaboration; 2010. p. 1-61.

21. Bossuyt P, Davenport C, Deeks J, Hyde C, Leeflang M, Scholten R. Chapter 11. Interpreting results and drawing conclusions. In: Deeks J, Bossuyt $P$, Constantine G, editors. Cochrane Handbook for Systematic Reviews of Diagnostic Test Accuracy Version 0. ed: The Cochrane Collaboration; 2013.

22. Hajian-Tilaki K. Receiver operating characteristic (ROC) curve analysis for medical diagnostic test evaluation. Caspian Journal of Internal Medicine. 2013:4(2):627-35.

23. Deeks JJ, Wisniewski S, Davenport C. Chapter 4: Guide to the contents of a Cochrane Diagnostic Test Accuracy Protocol. In: Deeks JJ, Bossuyt PM, Gatsonis C, editors.: The Cochrane Collaboration; 2013. p. 1-15.

24. van Enst WA, Ochodo E, Scholten RJPM, Hooft L, Leeflang MM. Investigation of publication bias in meta-analyses of diagnostic test accuracy: a metaepidemiological study. BMC Med Res Methodol. 2014;14:70.

25. Liberati A, Altman DG, Tetzlaff J, Mulrow C, Gøtzsche PC, John PA. The PRISMA statement for reporting systematic reviews and meta-analyses of studies that evaluate healthcare interventions explanation and elaboration. BMJ. 2009;339:b2700.

26. Farb HF, Arnesen M, Geistler P, Knox GE. C-reactive protein with premature rupture of membranes and premature labor. Obstet Gynecol. 1983;62(1):49-51.

27. Hawrylyshyn P, Milligan JE, Soldin S, Pollard A, Papsin FRP. Bernstein Premature rupture of membranes: the role of C-reactive protein in the prediction of chorioamnionitis. Am J Obstet Gynecol. 1983;147(3):240-6.
28. Ismail MA, Zinaman MJ, Lowensohn Rl, Moawad AH. The significance of Creactive protein levels in women with premature rupture of membranes. Am J Obstet Gynecol. 1985;151(4):541-4.

29. Fisk J, Child AG, Gatenby PA, Jeffery H, Bradfield AHNM. Fysh. Is C-reactive protein really useful in preterm premature rupture of the membranes? $\mathrm{Br}$ J Obstet Gynaecol. 1987;94(12):1159-64.

30. Danielian PJ. CA 125 and preterm prelabour rupture of the membranes. Br J Obstet Gynaecol. 1991;98(8):835-6.

31. Yoon BH, Jun JK, Park KH, Syn HC, Gomez R, Romero R. Serum C-reactive protein, white blood cell count, and amniotic fluid white blood cell count in women with preterm premature rupture of membranes. Obstet Gynecol. 1996;88(6):1034-40.

32. Torbe A. Maternal plasma procalcitonin concentrations in pregnancy complicated by preterm premature rupture of membranes. Mediat Inflamm. 2007;2007:35782.

33. Murtha AP, Sinclair T, Hauser ER, Swamy GK, Herbert WNP, Heine RP. Maternal serum cytokines in preterm premature rupture of membranes. Obstet Gynecol. 2007;109(1):121-7.

34. Smith CL, Sartorius JA, White DR, Maslow ASEJ. Muller. C-reactive protein as a predictor of chorioamnionitis. J Am Osteopath Assoc. 2012;112(10):660-4.

35. Perrone G, Anceschi MM, Capri O, Galoppi P, Pizzulo S, Buccheri M, et al. Maternal C-reactive protein at hospital admission is a simple predictor of funisitis in preterm premature rupture of membranes. Gynecol Obstet Investig. 2012;74(2):95-9.

36. Gulati S, Agrawal S, Raghunandan C, Bhattacharya J, Saili A, Agarwal S, et al. Maternal serum interleukin-6 and its association with clinicopathological infectious morbidity in preterm premature rupture of membranes: a prospective cohort study. J Matern Fetal Neonatal Med. 2012;25(8):1428-32.

37. Gulati S, Bhatnagar S, Raghunandan C, Bhattacharjee J. Interleukin-6 as a predictor of subclinical chorioamnionitis in preterm premature rupture of membranes. Am J Reprod Immunol. 2012;67(3):235-40.

38. Canzoneri BJ, Grotegut CA, Swamy GK, Brancazio LR, Sinclair T, Heine PR, et al. Maternal serum interleukin-6 levels predict impending funisitis in preterm premature rupture of membranes after completion of antibiotics. J Matern Fetal Neonatal Med. 2012;25(8):1329-32.

39. Oludag F, Caglayan E, Saatli B, Okyay RE, Altunyurt ST. Gode. Value of maternal procalcitonin levels for predicting subclinical intra-amniotic infection in preterm premature rupture of membranes. J Obstet Gynaecol Res. 2014;40(4):954-60.

40. Aksakal O, Altinbas S, Esin S, Muftuoglu KHSE. Kandemir. Fetal tyhmus size as a predictor of histological chorioamnionitis in preterm premature rupture of membranes. J Matern Fetal Neonatal Med. 2014;27(11):1118-22.

41. Ronzino-Dubost V, Sananes N, Lavaux T, Youssef C, Gaudineau A, Lecointre $L$, et al. Evaluation of the interest of procalcitonin in the diagnosis of chorioamnionitis in preterm premature rupture of membranes. An observational and prospective study. Journal de Gynecologie Obstetrique et Biologie de la Reproduction. 2016;45(7):745-53.

42. Thornburg LL, Queenan R, Brandt-Griffith B, Pressman EK. Procalcitonin for prediction of chorioamnionitis in preterm premature rupture of membranes. The journal of maternal-fetal \& neonatal medicine : the official journal of the European Association of Perinatal Medicine, the Federation of Asia and Oceania Perinatal Societies, the International Society of Perinatal Obstetricians. 2016;29(13):2056-61.

43. Kim SA, Park KH, Lee SM. Non-invasive prediction of histologic chorioamnionitis in women with preterm premature rupture of membranes. Yonsei Med J. 2016;57(2):461-8.

44. Stepan M, Cobo T, Musilova I, Hornychova H, Jacobsson B, Kacerovsky M. Maternal serum C-reactive protein in women with preterm prelabor rupture of membranes. PLoS One. 2016;11(3):e0150217.

45. Kayem G, Batteux F, Girard N, Schmitz T, Willaime M, Maillard F, et al. Predictive value of vaginal IL-6 and TNFalpha bedside tests repeated until delivery for the prediction of maternal-fetal infection in cases of premature rupture of membranes. Eur J Obstet Gynecol Reprod Biol. 2017:211:8-14.

46. Broumand F, Naji S, Seivani S. Predictive values of maternal serum levels of procalcitonin, ESR, CRP, and WBC in the diagnosis of chorioamnionitis in mothers with preterm premature rupture of membrane. Iranian Journal of Neonatology. 2018;9(2):50-60.

47. Seivani S, Broumand F, Naji S. Predictive value of maternal serum level of procalcitonin in diagnosing chorioamnionitis in mothers with preterm 
premature rupture of membrane (PROM). Internal Medicine and Medical Investigation Journal. 2017;2:4.

48. Martinez-Portilla RJ, Hawkins-Villarreal A, Alvarez-Ponce P, Chinolla-Arellano ZL, Moreno-Espinosa AL, Sandoval-Mejia AL, et al. Maternal serum interleukin-6: a non-invasive predictor of histological chorioamnionitis in women with preterm-prelabor rupture of membranes. Fetal Diagn Ther. 2019;45(3):168-75.

49. Asadi N, Faraji A, Keshavarzi A, Akbarzadeh-Jahromi M, Yoosefi S. Predictive value of procalcitonin, C-reactive protein, and white blood cells for chorioamnionitis among women with preterm premature rupture of membranes. International journal of gynaecology and obstetrics: the official organ of the International Federation of Gynaecology and Obstetrics. 2019; 147(1):83-8.

50. Park JW, Park KH, Lee JE, Kim YM, Lee SJ, Cheon DH. Antibody microarray analysis of plasma proteins for the prediction of histologic chorioamnionitis in women with preterm premature rupture of membranes. Reprod Sci. 2019.

51. de Vet H, Eisinga A, Riphagen I, Aertgeerts B, Pewsner D. Chapter 7: Searching for studies. Cochrane handbook for systematic reviews of diagnostic test accuracy The Cochrane Collaboration; 2008.

52. Schmidt RL, Factor RE. Understanding sources of bias in diagnostic accuracy studies. Arch Pathol Lab Med. 2013;137(4):558-65.

53. Cobo B, Kacerovsky M, Hougaard DM, Skogstrand K, Gratacos E, Palacio MT, et al. Systemic and local inflammatory response in women with preterm prelabor rupture of membranes. PLOS ONE. 2014;9(1):e85277-e.

54. Bossuyt PM, Reitsma JB, Bruns DE, Gatsonis CA, Glasziou PP, Irwig LM, et al. The STARD statement for reporting studies of diagnostic accuracy: explanation and elaboration. Ann Intern Med. 2003;138(1):W1-12.

\section{Publisher's Note}

Springer Nature remains neutral with regard to jurisdictional claims in published maps and institutional affiliations.

Ready to submit your research? Choose BMC and benefit from:

- fast, convenient online submission

- thorough peer review by experienced researchers in your field

- rapid publication on acceptance

- support for research data, including large and complex data types

- gold Open Access which fosters wider collaboration and increased citations

- maximum visibility for your research: over $100 \mathrm{M}$ website views per year

At $\mathrm{BMC}$, research is always in progress.

Learn more biomedcentral.com/submissions 